Published by Al-Nahrain College of Medicine P-ISSN 1681-6579

E-ISSN 2224-4719

Email: iraqijms@colmed-alnahrain.edu.iq http://www.colmed-alnahrain.edu.iq http://www.iraqijms.net

\title{
Standard Disscectomy versus Microdiscectomy: Short Term and Long Term Outcome Comparison in Treatment of Lateral Lumbar Disc Hernaiation
}

\author{
Mohamed A. Al-Tamimi FICMS \\ Dept. of Surgery, College of Medicine, Al-Mustansiriya University, Baghdad, Iraq
}

\author{
Abstract \\ Background
}

Objective

Methods

Results

Conclusion

Keywords

DOI: $10.22578 /$ IJMS.14.4.10

List of abbreviation: $L B P=$ Low back pain
Despite the high incidence of coincident spinal degenerative changes due to the high dynamic interplay between adjacent spinal elements leading to the clinical pain syndromes, yet the diagnostic approach and therapeutic options are still diverse and often inconsistent.

To evaluate the short and long term outcome of two different surgical approaches in the treatment of lateral lumbar disc prolapse associated with spondylosis.

Twenty patients presenting with a comparable complaints of radicular low back pain falling in the age group of 40-50 who attended the outpatient clinic in Science and Technology Hospital in Sanaa from 1st January 2008 to 1st of June 2009 and who were diagnosed to have lumbar lateral disc prolapse with mild spondylotic changes in need for surgery were divided into two groups. Group A offered microdiscectomy while group B offered standard discectomy. They were followed up and evaluated both clinically and radiologically at fixed postoperative intervals (day of discharge, three months, and one year post operatively).

It has been revealed that most of cases showed improvement of their presenting complaints due to the acute decompression offered to the neural tissue by either approach though was initially much higher with the minimally invasive microdiscectomy. However, the picture changed at three months interval where (30\%) of patients from group A had complaints, two cases (66.6\%) of the incompletely responding cases presented with new symptoms mostly due to incompletely treated spondylotic changes and 1 patient (33.3\%) of the incompletely responding cases presented with persistence of symptoms due to incomplete disc removal. In contrast, only one case from group $B$ had the persistence of symptoms, which was due to the effect of spondylosis. With further follow up at 1 year interval $40 \%$ of cases from group $A$ had complaints mostly in form of bilateral radiating pain due to incompletely treated spondylotic changes, while only (20\%) from group B had complaints either as ipsilaterally radiating pain due to incomplete disc resection or as bilaterally radiating pain due to postoperative adhesions.

Treatment with the first modality though has the advantages of a shorter duration of surgery, less invasion, less postoperative stay at hospital and comparable clinical response on short term follow up to that of second group, yet data at long term follow up showed that it is associated with a higher incidence of recurrence or incomplete resolve of the presenting complaint as well as evident evolving radiological complications in contrast to treatment by the second modality. Lateral disc prolapse, spondylosis, micro discectomy, standard discectomy.

\section{Introduction \\ T ow back pain (LBP) affects approximately $60-85 \%$ of adults during some point in their lives ${ }^{(1-3)}$. Fortunately, for the large}


majority of individuals, symptoms are mild and transient, with 90\% subsiding within 6 weeks (4). Chronic back pain which is defined as pain symptoms persisting beyond 3 months affects an estimated $15-45 \%$ of the population ${ }^{(5,6)}$ is particularly important being responsible for completely disturbing patient's day activities with subsequent burdens on a family and community basis ${ }^{(7)}$. Lateral disc herniations as one of the causes of back pain constitute 7$12 \%$ of all disc herniations. They may be purely far lateral or extraforaminal in location, located beyond the pedicles, or may include intraforaminal and even intracanalicular components. Lateral disc herniations are occurring predominantly at the L4-L5 and L3-L4 levels in almost equal numbers. Clinical syndromes reflect compression of the superiorly exiting nerve root and ganglion; i.e. a L4-L5 far lateral disc produces a L4 root syndrome ${ }^{(8)}$. Intervertebral discs are believed to undergo what Kirkaldy Willis and Bernard (9) first coined a "degenerative cascade" of three overlapping phases that may occur over the course of decades. Phase I (Dysfunction Phase), Phase II (Instability Phase) ${ }^{(10)}$ and Phase III (Stabilization Phase) (11). On the other hand, spondylosis of the lumbar spine is considered mechanically, as the hypertrophic response of adjacent vertebral bone to disc degeneration (although osteophytes may infrequently form in the absence of diseased discs) ${ }^{(13)}$. In another word, spondylosis may be applied nonspecifically to all degenerative conditions affecting the discs, vertebral bodies, and/or associated joints of the lumbar spine ${ }^{(12,13)}$. For purposes of this review, we will use this final, broad definition of spondylosis, recognizing the high incidence of coincident degenerative changes, and the dynamic interplay between adjacent discs, vertebra, and nerves that create the clinical pain syndromes within the axial spine and associated nerves. Elective lumbar discectomy is regarded as a good treatment option for lumbar disc herniation if sciatica or neurological deficits occur and still persist after 6 weeks of conservative therapy ${ }^{(14-16)}$. Mixter and Barr first described herniated disc as a cause of neural compression in the lumbar spinal canal in $1934^{(17)}$. They described a surgical approach to the problem that involved partial hemilaminectomy and partial removal of the disc (standard open discectomy). In 1977, a new technology was introduced by Yasargil ${ }^{(18)}$ and Caspar ${ }^{(19)}$ that involved the use of an operating microscope for the surgical removal of the disc. They independently described microsurgical techniques that provided excellent lighting and magnification of the operative field. Compared with the standard open discectomy, the microdiscectomy enabled the use of smaller incisions of the skin and fascia and facilitated a less traumatic surgical procedure. The first follow-up report of Williams et al. in 1978 showed encouraging results following lumbar micro discectomy ${ }^{(20)}$. Since that time, these two procedures have been considered the gold standard for the surgical treatment of lumbar disc herniations. However, there is a little consensus with regard to a definitive treatment approach for disc herniation associated with spondylosis and seldom comparative studies focusing on the short and long term outcome have been attempted. Thus, the current study was conducted to evaluate the short and long term outcome for standard and micro discectomy procedures in the treatment of symptomatic lateral lumbar disc prolapse associated with mild degree of spondylosis.

\section{Methods}

This is a descriptive study comparing the short and long term outcome of 2 different surgical approaches in the treatment of lateral lumbar disc prolapse associated with spondylosis, using simple statistical measures in the description as numbers and percentages. The samples were included twenty patients of both sexes falling in the age group of 40-50 years, who attended to the outpatient clinic in the University of Science and Technology Hospital in Sanaa from $1^{\text {st }}$ January 2008 to $1^{\text {st }}$ of June 2009. The patients participated in the study were examined clinically and radiologically 
preoperatively and were diagnosed to be in need for surgical treatment. An informed consent was taken from all the patients to participate in this study.

\section{Patient's inclusion criteria were as follows:}

1. All of the patients had a comparable affected level of activities according to Oswestry Low Back Pain Scale.

2. All of the patients had a comparable intensity of back and radicular pain according to Oswestry Low Back Pain Scale. In which, all of them had clinical complaints including moderate to severe radicular pain accompanied by very positive mechanical signs; LaseÂgue and reverse LaseÂgue (femoral stretch test) maneuvers. Neurological deficit signs and symptoms including motor, reflex, and sensory findings were consistent with their radiological findings.

3. Radiologially, all of them having the diagnosis of lateral disc prolapse and mild degree of spondylosis in the lumbar vertebrae.

4. All of the patients were operated by the same surgeon.

All the patients who had similar complaints due to other pathologies or had not been subjected to accurate conservative treatment or had previous back surgical interventions were excluded.

For the purpose of this study, the patients were allocated into two groups each of ten:

1. Group A: patients were operated by unilateral microdiscectomy.

2. Group B: patients were operated by standard discectomy which includes: hemilaminectomy and total ligamentum flavum resection and bilateral foraminotomy.

After surgery the patients were followed up by the researcher himself (specialist neurosurgeon) both clinically at fixed postoperative intervals (day of discharge, three months, and one year postoperative) and radiologically at 3 months and 12 months interval. These follow up dates are set with the patients before the operation as part of the treatment strategy in the department. For aim of clarification, we will use the term incompletely responding cases to those who either did not show clinical response to surgical treatment or had improvement and then recurrence of complaint or had newly evolving but related complaints.

\section{Results}

Our study showed that, with regard to clinical response in terms of resolve of the presenting chief complaint, the majority of patients in both groups showed response (clinically and with the aid of Oswestry Low Back Pain Scale) at the three follow up intervals as shown in figure 1.

With regard to incompletely responding cases they were further analyzed at 3 months and 12 months postoperative date.

First of all, according to their clinical picture (through reviewing their signs and symptoms with the aid of Ostwestry back pain scale to assess their pain intensity and site and improvement together with clinical examination):

At three months postoperative date, the incompletely responding cases from group $A$ fell in three different clinical presentations in equal percentages which was either radiating pain at similar preoperative side or on contralateral side or bilaterally radiating, while the only incompletely responding case in group $B$ fell in the ipsilateral radicular pain sector as shown in table 1.

At 1 year postoperative follow up date other cases were added to the incompletely responding sector of both groups (emphasizing that those who showed incomplete response at 3 months follow up date were still showing no further response at 1 year postoperative period). The additional two incompletely responding cases (one from group $A$ and another one from group B) fell into the category of bilaterally radiating lower back pain (stressing that one incompletely responding case from group B showed resolve of complaint) as apparent in table 2. 


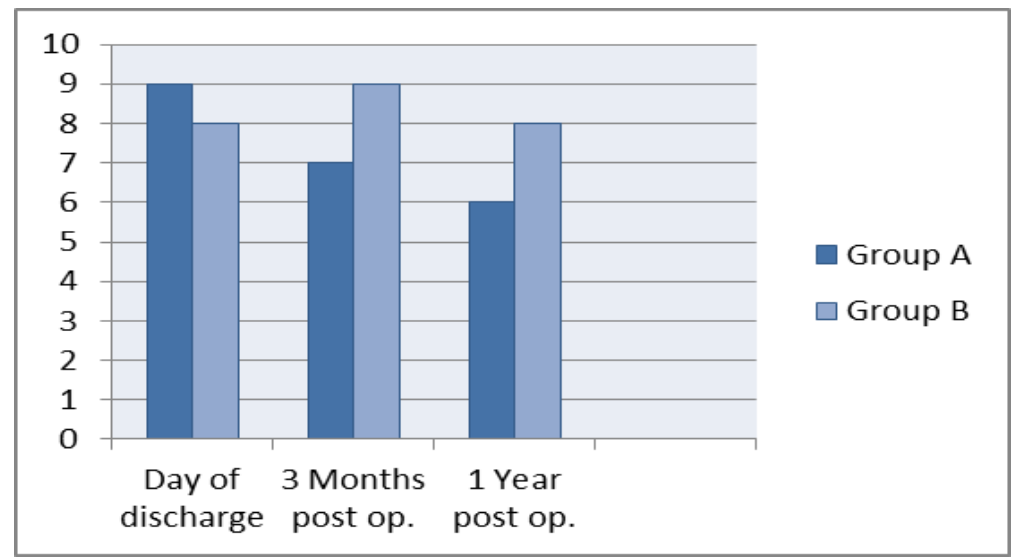

Figure1: Histogram showing the relationship between the type of surgery and pain resolution at three time intervals of follow up

Table 1. Distribution of the incompletely responding cases of both groups in respect to clinical picture 3 months postoperatively

\begin{tabular}{ccccc}
\hline \multirow{2}{*}{ Clinical complaints } & \multicolumn{2}{c}{ Group A } & \multicolumn{2}{c}{ Group B } \\
& No. & $\%$ & No. & $\%$ \\
\hline Pain at similar side & 1 & $33.3 \%$ & 1 & $100 \%$ \\
Pain on contralateral side & 1 & $33.3 \%$ & 0 & $0 \%$ \\
Bilateral pain & 1 & $33.3 \%$ & 0 & $0 \%$ \\
\hline Total & 3 & $100 \%$ & 1 & $100 \%$ \\
\hline
\end{tabular}

Table 2. Distribution of the incompletely responding cases of both groups in respect to clinical picture one year postoperatively

\begin{tabular}{ccccc}
\hline \multirow{2}{*}{ Clinical complaints } & \multicolumn{2}{c}{ Group A } & \multicolumn{2}{c}{ Group B } \\
& No. & $\%$ & No. & $\%$ \\
\hline Pain at similar side & 1 & $25 \%$ & 1 & $50 \%$ \\
Pain on contralateral side & 1 & $25 \%$ & 0 & $0 \%$ \\
Bilateral pain & 2 & $50 \%$ & 1 & $50 \%$ \\
\hline Total & 4 & $100 \%$ & 2 & $100 \%$ \\
\hline
\end{tabular}

Thereafter, the patients were analyzed according to their relevant radiological findings through obtaining MRI to the lumbar spine with emphasis on the operation site and comparing that with the preoperative images: At three months postoperative date, the incompletely responding cases from group $A$ were mostly having progressive spondylotic changes with an effect on the nearby neural tissue (66.6\%) and to a lesser extent the presence of incomplete disc removal was evident in the other case (33.3\%). In group $B$ the MRI finding for the still incompletely responding case was that of spondylosis $(100 \%)$ as shown in table 3. 
Table 3. Distribution of incompletely responding cases of both groups in respect to MRI findings three months postoperatively

\begin{tabular}{ccccc}
\hline \multirow{2}{*}{ Clinical complaints } & \multicolumn{2}{c}{ Group A } & \multicolumn{2}{c}{ Group B } \\
& No. & $\%$ & No. & $\%$ \\
\hline Spondylosis & 2 & $66.7 \%$ & 1 & $100 \%$ \\
Incomplete disc removal & 1 & $33.3 \%$ & 0 & $0 \%$ \\
\hline Total & 3 & $100 \%$ & 1 & $100 \%$ \\
\hline
\end{tabular}

At 1 year, postoperative follow up date MRI picture of the incompletely responding cases in group A were that of spondylotic changes effect (50\%), the presence of Incomplete disc removal (25\%) and contralateral disc bulge (25\%), while the picture in group B was different with a spondylosis (50\%) and adhesions (50\%) as shown in table 3.
It is worthy to mention that the clinical picture of the responding cases in both groups was stable throughout the follow up period and their MRI picture did not show significant changes just like those in the incompletely responding group.

Table 4. Distribution of incompletely responding cases of both groups in respect to MRI findings one year postoperatively

\begin{tabular}{ccccc}
\hline \multirow{2}{*}{ Clinical complaints } & \multicolumn{2}{c}{ Group A } & \multicolumn{2}{c}{ Group B } \\
& No. & $\%$ & No. & $\%$ \\
\hline Spondylosis & 2 & $50 \%$ & 1 & $50 \%$ \\
Adhesions & 0 & $0 \%$ & 1 & $50 \%$ \\
Contralateral disc bulge & 1 & $25 \%$ & 0 & $0 \%$ \\
Incomplete disc removal & 1 & $25 \%$ & 0 & $0 \%$ \\
\hline Total & 4 & $100 \%$ & 2 & $100 \%$ \\
\hline
\end{tabular}

\section{Discussion}

In this study, it has been revealed that although at time of discharge the majority of patients in both groups showed improvement regarding their chief complaints, which can be attributed to relief of the neural tissue compression offered by surgery with either modality, however, three months later, the picture was changed in such a way that certain number of patients in group $A$ showed either recurrence or newly evolving related symptoms, in contrast to group B where the number of the responding cases raised up. This could be explained by reviewing the radiological findings for the incompletely responding cases in both groups in which, 2 cases out of the 3 incompletely responding cases within group A showed the picture of persistent not treated well nearby spondylotic degenerative changes that usually accompany the disc herniation process and thus persistent some degree of neural tissue compression, which was the cause for patients complaints in the form of contralaterally and bilaterally radiating back pain. Furthermore, one case out of the three incompletely responding cases showed persistent ipsilaterally radiating back pain due to the incomplete herniated disc removal. These results were the fact that limited access micro discectomy offered only 
partial removal of the herniated disc giving a temporary relief of symptoms but much less access to treat the associated spondylotic changes and suboptimum treatment for the herniated disc. While the only non-responding case in group $B$ was due to the progressive spondylotic changes. This disagrees with a study held by Porchet et al. ${ }^{(21)}$, who claimed that there was no difference between the classical macroscopic approach to lumbar disc herniation and the more modern micro discectomy.

On the other hand, it is believed that the cause behind the crescendo improvement in group $B$ was due to the more extensive decompression offered for the compressed neural elements by the second approach through extensive removal of the herniated disc together with the associated compromising osteophytes and the thickened ligamentum flavum and the better access to neural foramina bilaterally, that was clear in the postoperative MRI pictures of those patients as compared to the MRI findings in group A. When additional analyses were carried out to examine whether outcome could change over 1 year period of follow up, it was obvious that additional number of patients in both groups had recurrence of symptoms especially in Group A were the symptoms mostly were bilateral due to incomplete and suboptimum neural tissue freeing from the associated spondylotic changes whether at the side of the surgery or on the contralateral side and the development of a contralateral disc bulge with their effect collectively on the nearby neural tissue, yet none was attributed to postoperative adhesions as revealed in the postoperative MRI finding of this group and this is the usual occurrence with microdiscectomy were the wound is small and the postoperative adhesions are less. In contrast with the ordinary discectomy where the chance to remove all the causative pathological factors is much higher and more optimum due to the wide field offered by that approach even with the higher chance to get adhesions still the number of the responding cases is higher and the main cause between the incompletely responding cases was distributed between ongoing Spondylotic changes and postoperative adhesions ${ }^{(22)}$. As the vast majority of patients undergoing disc surgery in our Spine Centre do so in connection with degenerative disorders (spondylosis), it is obvious from all above that the early result was highly predictive of the longer-term outcome. This has been reported before, in relation to surgery for degenerative diseases of the lumbar spine (23-26), and as surgery typically serves a "mechanical" purpose, aiming to relieve pain by removing all the causes of physical obstruction including disc and associated spondylotic effects we can see that the limited access micro discectomy was deficient in this aspect in comparison with ordinary discectomy.

This study concluded that treatment with the first modality though has the advantages of a shorter duration of surgery, less invasion, less postoperative stay at hospital and comparable clinical response on short term follow up to that of second group, yet data at long term follow up show that it is associated with a higher percentage of recurrence or incomplete resolve of the presenting complaint as well evolving radiological complications. On the other hand, patients treated with the more extensive surgery, despite having the draw backs of a longer duration of surgery, being more extensively invasive, and requiring a relative longer postoperative stay at hospital, however, they had their preoperative complaints resolving, more rapidly than in the $1^{\text {st }}$ group and the resolution seeming to be more permanent with less radiological complication on long term basis.

Therefore, our recommendations are that well patients with longer life expectancy, patients without medical diseases that stand against longer period of anesthesia to be operated on by the second more extensive approaches (without disturbing the stability) for longer lasting and better outcome. Anyhow, this is a 
descriptive study of observations from our practice, and we do recommend further analytic studies in this respect.

\section{Acknowledgments}

Great thanks to all participants in this study specially the managers and for all Neurosurgery Unit Staff in the University of Science and Technology Hospital in Sanaa, Yemen.

\section{Conflict of interest}

The author has no conflicts of interest.

\section{Funding}

No special funds required for this study.

\section{References}

1. Frymoyer JW. Back pain and sciatica. N Engl J Med. 1988; 318: 291-300.

2. Van Geen J, Edelaar $M$, Janssen $M$, et al. The longterm effect of multidisciplinary back training: a systematic review. Spine. 2007; 32(2): 249-55.

3. Andersson GB. Epidemiological features of chronic low pain. Lancet. 1999; 354: 581-5.

4. Dillane J, Fry J, Kalton G. Acute back syndrome - a study from general practice. Br Med J. 1966; 2: 82-4.

5. Andersson $\mathrm{HI}$, Ejlertsson $\mathrm{G}$, Leden I, et al. Chronic pain in a geographically defined general population: Studies of differences in age, gender, social class and pain localization. Clin J Pain. 1993; 9: 174-82.

6. Andersson GB. The epidemiology of spinal disorders. In: Frymoyer JW. (ed.) The adult spine: principles and practice. 2nd ed. Philadelphia, PA: Lippincott-Raven; 1997.

7. van Tulder MW, Koes BW, Bouter LM. A cost-ofillness study of back pain in The Netherlands. Pain. 1995; 62: 233-40.

8. Epstein NE. Foraminal and far lateral lumbar disc herniations: surgical alternatives and outcome measures. Spinal Cord. 2002: 40, 491-500.

9. Middleton K, Fish DE. Lumbar spondylosis: Clinical presentation and treatment approaches. Curr Rev Musculoskeletal Med. 2009; 2: 94-104.

10. Boswell MV, Trescot AM, Datta S, et al. Interventional techniques: evidence-based practice guidelines in the management of chronic spinal pain. Pain Physician. 2007; 10(1): 7-111.

11. Kirkaldy-Willis $\mathrm{WH}$, Wedge $\mathrm{JH}$, Yong-Hing $\mathrm{K}$, et al. Pathology and pathogenesis of lumbar spondylosis and stenosis. Spine. 1978; 3: 319-28.
12. Schneck CD. The anatomy of lumbar spondylosis. Clin Orthop Relat Res. 1985; 193: 20-36.

13. Gibson JNA, Waddell G. Surgery for degenerative lumbar spondylosis. Spine. 2005; 20: 2312-20.

14. Deyo RA. Back surgery-who needs it? N Engl J Med. 2007; 356: 2239-43.

15. Gibson JN, Waddell G. Surgical interventions for lumbar disc prolapse: updated Cochrane Review. Spine. 2007; 32: 1735-47.

16. Peul WC, van Houwelingen $\mathrm{HC}$, van den Hout WB, et al. Surgery versus prolonged conservative treatment for sciatica. N Engl J Med. 2007; 356: 2245-56.

17. Mixter WJ, Barr JS. Rupture of the intervertebral disc with involvement of the spinal canal. N Engl J Med. 1934; 211: 210-25.

18. Yasargil MG. Microsurgical operation for herniated disc. Adv Neurosurg. 1977; 4: 81.

19. Caspar W. A new surgical procedure for lumbar disc herniation causing less tissue damage through a microsurgical approach. Adv Neurosurg 1977; 4: 7480.

20. Williams RW. Microlumbar discectomy: A conservative surgical approach to the virgin herniated lumbar disc. Spine 1978; 3: 175-82.

21. Porchet F, Bartanusz V, Kleinstueck $S$, et al. Microdiscectomy compared with standard discectomy: an old problem revisited with new outcome measures within the framework of a spine surgical registry. Eur Spine J. 2009; 18(Suppl 3): 3606.

22. Fritsch EW, Heisel J, Rupp S. The Failed Back Surgery Syndrome. Spine. 1996; 21(5): 626-33.

23. Amundsen $T$, Weber $H$, Nordal $H J$, et al. Lumbar spinal stenosis: conservative or surgical management? a prospective 10-year study. Spine. 2000; 25: 1424-35.

24. Hakkinen $A$, Ylinen J, Kautiainen $H$, et al. Does the outcome 2 months after lumbar disc surgery predict the outcome 12 months later? Disabil Rehabil. 2003; 25: 968-72.

25. Mannion AF, Elfering A. Predictors of surgical outcome and their assessment. Eur Spine J. 2006; 15(Suppl 1): S93-S108.

26. McGregor $A H$, Hughes SPF. The evaluation of the surgical management of nerve root compression in patients with low back pain. Part 1: The assessment of outcome. Spine. 2002; 27: 1465-70.

E-mail: mhmdtamimi88@yahoo.com
Received $1^{\text {st }}$ Sep. 2016: Accepted $26^{\text {th }}$ Oct.
2016

